\title{
CRS as a tool for true amplitude imaging
}

\author{
R. S. Portugal, R. Biloti, L. T. Santos and M. Tygel
}

State University of Campinas, Brazil

\section{Abstract}

We present a method to obtain a true-amplitude migration and amplitude-versus-angle (AVA) at selected points using the attributes generated by the Common Reflection Surface (CRS) Stack. Our approach combines the CRS stack/inversion process applied to multicoverage data, together with the use of a kinematic Kirchhoff migration, to achieve true-amplitudes (TA) at assigned depth points of the migrated images. The proposed method consists of the following steps: (i) apply the CRS process to the given multicoverage data; the obtained CRS attributes are next used to produce a simple macro-velocity depth model; (ii) perform an unweighted Kirchhoff migration for imaging purposes only; for selected points on target reflectors in the migrated image, we use the macro-velocity model to determine, by ray tracing, common-reflectionpoint (CRP) gathers that belong to the input data; for these rays, we compute the incident angles and the geometrical spreadings; (iii) go back to CRP gathers and compensate the amplitudes for geometrical spreading. The results permit to construct AVA curves on the assigned CRPs. In summary, our method is designed to aggregate amplitude information on selected points of a reflector, after a purely kinematic image (migration) has been obtained. The method is tested on a synthetic inhomogeneous layered model with good results.

\section{Introduction}

One of the main objectives of processing seismic reflection data for hydrocarbon prospecting is to obtain meaningful images of the geological structures, in particular reservoir structures in the subsurface. The geological structures to be imaged are defined by seismic reflectors.

Kinematical images, in which only the location and orientation of the reflectors (with no regard to amplitudes) are considered, can be achieved, for example, by efficient Kirchhoff migration procedures using simple weights or no weights at all. Kirchhoff migration requires a given macrovelocity model. Moreover, special methods exist to combine the migration outputs to update the model, so as to refine and improve the image. The final result is, in many cases, a fairly adequate (kinematical) image of the structures of interest.

The problems that concern us in this paper is how to aggregate dynamical information (amplitudes) to the obtained image. In fact, the amplitudes are needed essentially on selected points at key interfaces, where the determination of angledependent reflection coefficients is the most desirable information.

According to zero-order ray theory, the amplitude of a primary-reflection event can be described by

$$
U=\mathcal{A} \frac{R_{c}}{\mathcal{L}},
$$

where $R_{c}$ is the angle-dependent reflection coefficient of the primary reflection ray and $\theta$ is the incidence angle of that ray with respect to the interface normal. The reflection coefficient is the quantity of interest to be estimated from the data. The quantity $\mathcal{L}$ is the angle-dependent geometrical-spreading factor of the reflection ray. It accounts for the amplitude variations due to focusing and defocusing of the energy carried by the ray along its ray path. All factors which affects amplitudes other than the geometrical spreading are combined and represented by overall quantity $\mathcal{A}$ in equation (1). The geometrical spreading, $\mathcal{L}$, is generally singled out as one of the major sources of amplitude distortion in the observed data. That is the reason why the term true-amplitude is typically attached to a primaryreflection amplitude that has been corrected for geometrical spreading.

In the case of depth migration, the term trueamplitude (TA) migration refers to the case in which the migration output equals the observed amplitudes automatically corrected for geometrical spreading (see, e.g., Hubral et al., 1996). Full TA algorithms are significantly more expensive and time-consuming than their kinematic unweighted counterparts. As another complication, the accuracy requirements on the macro-velocity depth model are higher for the application of TA migration than for purely kinematic migration. The flexibility of using migration outputs to update the velocity model is lost when such a heavy migration algorithm is applied. As a last, and probably the best, argument against the application of a full TA migration algorithm to an overall region is that, in fact, the amplitude information is required only 
Portugal et al.

on some specific target points or reflectors. Away from these points, the obtained amplitudes are not useful.

In this paper, we propose a method to aggregate true amplitudes (i.e., observed primaryreflection amplitudes after geometrical-spreading correction) at selected CRPs of interest, after an image of the subsurface has been obtained. This image can be, for example, the result of one or several kinematic migrations.

\section{Strategy}

The kernel of the method is summarized by the fluxogram shown in Figure 1. Our strategy is mainly divided in three steps: CRS attribute extraction \& macro-model inversion, kinematic imaging through unweighted Kirchhoff migrations and subsequent geometrical spreading corrections in the input data.

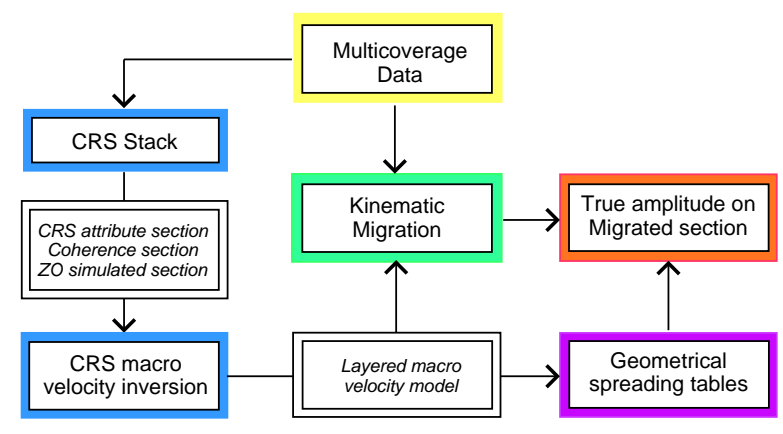

Figure 1: Fluxogram.

CRS stack. The 2-D common-reflection-surface (CRS) stack (see, e.g., Müller 1999), applied to multicoverage data on a seismic line, is designed to produce a stacked section (an approximation of a zero-offset section), together with three auxiliar sections of CRS attributes and a coherence section.

For each fixed central point (e.g., a CMP location of the original data), on which the output trace is to be computed, the CRS uses a multiparametric traveltime formula to stack all data that correspond to arbitrary source and receiver locations in the vicinity of that point. In this sense, it differs significantly from the conventional NMO/DMO stack (that employs only reflections from CMP gathers) to achieve much more redundancy with a consequent improvement of signal-to-noise ratio. The three CRS attributes assigned to each point of the stacked section are the parameters of the traveltime moveout formula. These are the emergence angle of the normal reflection ray and the wavefront curvatures of the NIP- and N-waves that arrive at that point. For the definition of the NIP-wave and the $\mathrm{N}$-wave, the reader is referred to the original paper of Hubral (1983).

The CRS attributes are extracted upon the use of coherency analysis strategy directly applied to the data. The determination of more efficient and accurate parameter extraction methods is a topic of active research (Birgin et al., 1999).

CRS macro-velocity model inversion. The philosophy of the CRS Stack method is to use as much data as possible during the stacking process. Therefore, the most relevant events are better defined on the stacked section and available for further inversion.

The input data for CRS velocity inversion are the CRS attributes that refer to those selected target reflections. Also the near-surface velocity field needs to be known. In fact, this is already a requirement for the application of the CRS method.

The classical layer-stripping velocity inversion algorithm of Hubral and Krey (1980) can be recast in terms use of the CRS, inverts iteratively on the depth the homogeneous layer velocities and the interface positions. The interfaces are constructed as cubic splines, which are suitable for further blocky ray tracing algorithms.

Kinematic image. As soon as the homogeneous layered velocity model is provided by the CRS inversion, it is smoothed in order to perform a kinematic migration. The traveltime tables are generated on the fly by the wavefront construction method, therefore each seismic trace can be migrated independently from each other. To enhance the signal-to-noise ratio, the final image is built by stacking all common-offset migrated section, this section is called stacked migrated section.

Geometrical spreading correction. On the stacked migrated section, it is possible to choose depth points on a target reflector. For each one chosen point, using the approximated homogeneous layered model, we compute, by standard dynamic ray-tracing, the traveltimes, the incident angles and the geometrical spreading factors. These quantities help to extract a common-reflection-point (CRP) gather from the original data. For each trace, using the computed reflection traveltime, we can pick 
CRS as a tool for true amplitude imaging

the amplitude, which is multiplied by the corresponding geometrical spreading factor. Applying this successively for all traces in the CRP gather, we obtain the desired AVO/AVA curves.

\section{Synthetic example}

The synthetic model, depicted in Figure 2, is composed by four layers separated by smooth interfaces. The first and fourth layers are homogeneous with constant compressional velocity of $2.0 \mathrm{~km} / \mathrm{s}$ and $2.7 \mathrm{~km} / \mathrm{s}$, respectively. The second and the

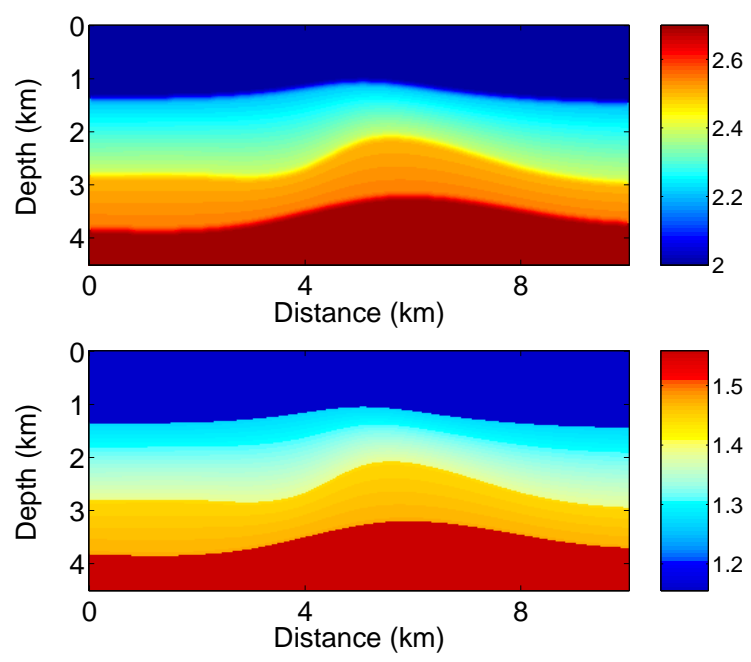

Figure 2: Velocity model for synthetic data. Top: compressional velocity. Bottom: shear velocity.

third layers are inhomogeneous, and their velocities are composed as a linear combination of the velocity just below the upper interface of the layer and the velocity just above the lower interface that bounds the layer. For those layers, the compressional velocity varies from $2.2 \mathrm{~km} / \mathrm{s}$ to $2.4 \mathrm{~km} / \mathrm{s}$ and from $2.5 \mathrm{~km} / \mathrm{s}$ to $2.55 \mathrm{~km} / \mathrm{s}$, respectively. The shear velocity in each point of the model is the compressional velocity divided by $\sqrt{3}$, and the density is unitary in the whole model. The multicoverage data is composed by 501 common-source experiments, where the sources are $20 \mathrm{~m}$ spaced. Each CS section has 151 receivers $20 \mathrm{~m}$ spaced. The ratio signal-to-noise in the data is 7:1. Figure 3 shows a typical common-offset section.

Each common-offset section was migrated separately, and then stacked to generate a stacked migrated section, depicted in Figure 4. The velocity model employed on the migration process (Figure 5) was obtained from the CRS attributes by

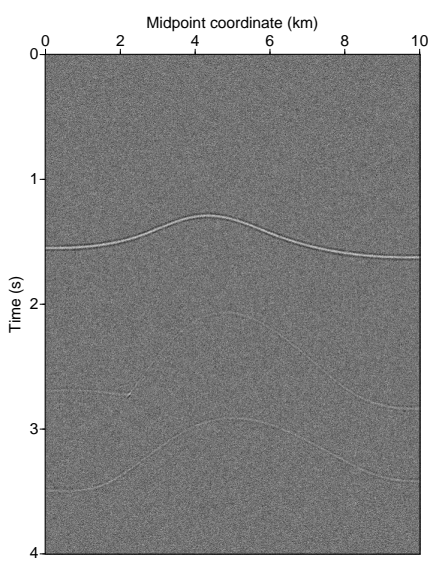

Figure 3: A typical common-offset section for the offset $1500 \mathrm{~m}$.

the inversion process briefly describe above. Note that the inverted model is as accurate as possible, since only homogeneous layers could be inverted.

Using this kinematic image we have chosen a point locate on the second interface to analyze the amplitude variation (AVO and AVA curves). Figures 6 and 7 show the CRP section and the AVO and AVA curves for the selected point, respectively.

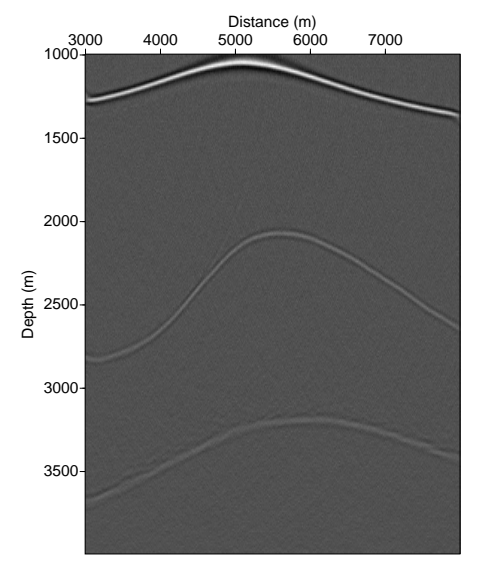

Figure 4: Stacked migrated section.

\section{Conclusions}

We have presented a method which provides a complete process to obtain AVA curves for chosen points on target interfaces. It is mainly composed by three steps: (i) construction of a layered macrovelocity model by using CRS attributes (obtained from the multicoverage data); (ii) kinematic migration of data using that macro-velocity model; (iii) $a$ 
Portugal et al.

posteriori correction of amplitude of chosen points on the migrated section (using traveltime, reflection angle and geometrical spreading computed on the approximated model). The numerical results are encouraging, concerning accurancy and computational effort. As a next step, further tests in real data will be carried out.

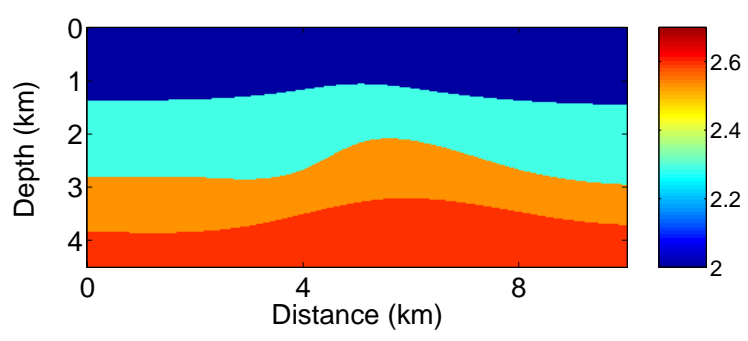

Figure 5: Compressional velocity model obtained by the CRS inversion algorithm.

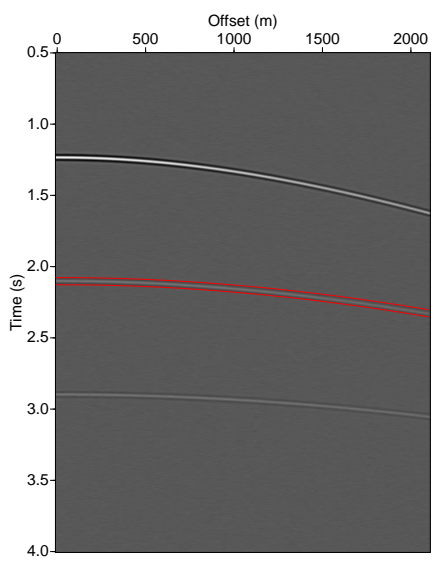

Figure 6: CRP section for the selected point on the second interface. The red tube confines the region where the picking process was carried out. This region was found out by the traveltime estimation that came out of the modelling process.

\section{Acknowledgments}

This work was partially supported by FAPESP (Grants 97/12125-8 and 97/12318-0) and by the sponsors of the WIT Consortium.
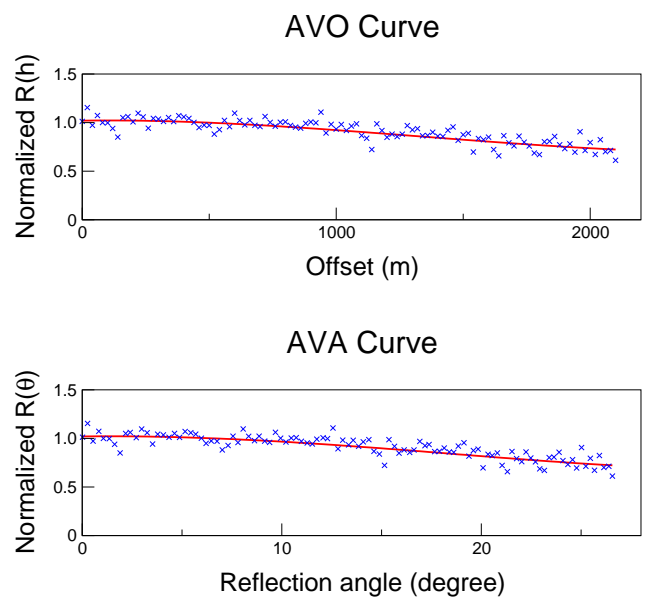

Figure 7: AVO and AVA curves for the chosen point on the second interface. The solid red line is the expected normalized reflection coefficient. The blue crosses are the picked amplitude correct for geometrical spreading, computed on the approximated model, and normalized.

\section{References}

Birgin, E. G., Biloti, R., Tygel, M., and Santos, L. T., 1999, Restricted optimization: a clue to a fast and accurate implementation of the common reflection surface method: Journal of Applied Geophysics, 42, 143-155.

Hubral, P., and Krey, T., 1980, Interval velocities from seismic reflection time measurements: Soc. of Expl. Geophys.

Hubral, P., Schleicher, J., and Tygel, M., 1996, A unified approach to 3-d seismic reflection imaging, part i: Basic concepts: Geophysics, 61, no. $03,742-758$.

Hubral, P., 1983, Computing true amplitude reflections in a laterally inhomogeneous earth: Geophysics, 48, no. 08, 1051-1062.

Müller, T., 1999, Common reflection surface stack method - seismic imaging without explicit knowledge of the velocity model: Ph.D. thesis, Geophysical Institute, Karlsruhe University, Germany. 